\title{
Efficiency of Nearest Neighbour Balanced Block Designs for Second Order Correlated Error Structure
}

\author{
R. Senthil kumar ${ }^{1}$ and C. Santharam ${ }^{2}$ \\ ${ }^{1}$ Assistant Professor, Department of Mathematics, Hindustan College of Arts and Science, Padur, \\ Chennai - 603103 (TN), INDIA \\ ${ }^{2}$ Rtd Professor, Department of Statistics, Loyola College, Chennai- 600034 (TN), INDIA
}

\begin{abstract}
The performance of a series of Complete and Incomplete Neighbour Balanced Block Designs for Auto Regressive (AR), Moving Average (MA) and Nearest Neighbour (NN) error correlation structure is studied when generalized least squares estimation is used. We have compared the efficiency of $A R(2), M A(2)$ and $N N$ correlation structures. It is observed that efficiency for direct as well as neighbour effects is high, in case of complete block designs for NN correlation structure. In case of incomplete block designs MA(2) correlation structure turns out to be more efficient as compared to others models with $\left(\rho_{1}, \rho_{2}\right)$ in the interval 0.1 to 0.4. It is therefore concluded that when block sizes are large and neighbouring plots are highly correlated, generalized least squares for estimation of direct and neighbour effects can be used. The gain in efficiency of NNBD and NNBIBD over regular block design is high under MA(2) models for direct and neighbour effects of treatments.
\end{abstract}

Keywords: Neighbour Balanced Block Design; Correlated observations; Generalized least squares; Auto Regressive; Moving Average; Nearest neighbour; Efficiency; Regular Block Design.

\section{Introduction}

In many experiments, especially in agriculture, the field on a given plot may be affected by treatments on neighbouring plots as well as by the treatment applied to that plot. To diminish these undesirable effects, Rees [15] first provided designs for the test and named such designs as neighbour designs. He used technique in virus research which requires arrangement in circles of samples from a number of virus preparations in such a way that over the whole set, a sample from each virus preparation appears next to a sample from every other virus preparation. He defined a neighbour design as an arrangement of $v$ antigens (called symbols) in $b$ circular plates (called blocks) such that: each block has $k$ symbols, not necessarily distinct, each symbol appears $r$ times in the design and each symbol is a neighbour of every other symbol precisely $\lambda$ times. A neighbour design with at least one block having less than $v$ distinct symbols may called as incomplete block neighbour designs. The designs in both series are neighbour balanced in the sense that every experimental treatment has each other treatment once as a right neighbour and once as a left neighbour. When treatments are varieties, neighbour effects may be caused by differences in height, root vigor, or germination date, especially on small plots, which are used in plant breeding experiments. Treatments such as fertilizer, irrigation, or pesticide may spread to adjacent plots causing neighbour effects. Such experiments exhibit neighbour effects, because the effect of having no treatment as a neighbour is different from the neighbour effects of any treatment. In case of block design setup if each block is a single line of plots and blocks are well separated, extra parameters are needed for the effect of left and right neighbours. An alternative is to have border plots on both ends of every block. Each border plots receives an experimental treatment, but it is not used for measuring the response variable. These border plots do not add too much to the cost of one-dimensional experiments. Neighbour balanced designs, where in the allocation of treatments is such that every treatment occurs equally often with every other treatment as neighbours, are used for these situations and permit the estimation of direct and neighbor effects of treatments. The neighbour effects are also called as interference effects, indirect effects or remote treatment effects.

The effect of correlation on the usual two-way analysis of variance and on the power of usual tests has been studied by Box [4]; Anderson et al. [1] and Aastveit [2]. Keedwell [9] considered 2-fold perfect circuit designs, these being balanced circuit designs whose neighbour properties apply not only to immediate neighbours but also to neighbours that are two places apart. In situations where the correlation structure among the observations within a block is known, may be from the data of past similar experiments, it may be advantageous to use this information in designing an experiment and analyzing the data so as to make more precise inference about treatment effects Gill and Shukla [6]. Neighbour balanced block designs for correlated errors by Kunert [10]. Lindner et al. [12] considered 2-perfect k-cycle systems of order $v$, i.e., balanced circuit RND's (Rees Neighbour Designs) whose neighbour properties hold both for immediate neighbours and for 2- 
places-apart neighbours. Optimal and highly efficient two dimensional designs have been constructed for correlated errors on the torus and in the two dimensional plane by Morgan and Nizamuddin [13]. Azais et al. [3] obtained a series of efficient neighbour designs with border plots that are balanced in $v-1$ blocks of size $v$ and $v$ blocks of size $v-1$, where $v$ is the number of treatments. Bailey [5] has given some designs for studying one-sided neighbour effects. These neighbour balanced block designs have been developed under the assumption that the observations within a block are uncorrelated. Kunert et al. [11] considered two related models for interference and have shown that optimal designs for one model can be obtained from optimal designs for the other model. Martin and Eccelston [14] have given variance balanced designs under interference and dependent observations. Santharam and Ponnuswamy [18] examined the optimality and efficiency of nearest neighbor balanced block designs when error follows Auto Regressive (AR), Moving Average (MA) or Auto Regressive Moving Average (ARMA) models. Senthil Kumar and Santharam [17] Efficiency of Nearest Neighbour Balanced Block Designs using ARMA models. Senthil Kumar and Santharam [16] Efficiency of NNBD over NNBIBD using First Order Correlated Models. Iqbal et al. [7] constructed second order neighbour designs for $3 \leq k \leq 7$ in circular block using method of cyclic shifts.

In this paper, neighbour balanced block designs for observations correlated within a block have been investigated for the estimation of direct as well as left and right neighbour effects of treatments. The performance of these designs for $\mathrm{AR}(2), \mathrm{MA}(2)$ and NN error correlation structure is studied when generalized least squares estimation is used. We have also investigated the efficiency of Nearest Neighbour Balanced Block Design (NNBD) and Nearest Neighbour Balanced Incomplete Block Design (NNBIBD) in comparison to regular block design when the error follows second order correlated models with $\left(\rho_{1}, \rho_{2}\right)$ in the interval $-0.4(-0.4) 0.4$.

\section{Model Structures and Information Matrix}

Let $\Delta$ be a class of binary neighbour balanced block designs with $n=b k$ units that form b blocks each containing k units. $Y_{i j}$ be the response from the $i^{\text {th }}$ plot in the $j^{\text {th }}$ block $(i=1,2, \ldots, k ; j=1,2, \ldots, b)$. The layout includes border plots at both ends of every block, i.e. at $0^{\text {th }}$ and $(k+1)^{\text {th }}$ position and observations for these units are not modeled. The following fixed effects additive model is considered for analyzing a neighbour balanced block design under correlated observations:

$$
Y_{i j}=\mu+\tau_{(i, j)}+l_{(i-1, j)}+\gamma_{(i+1, j)}+\beta_{j}+e_{i j}
$$

where $\mu$ is the general mean, $\tau_{(i, j)}$ is the direct effect of the treatment in the $i^{\text {th }}$ plot of $j^{\text {th }}$ block, $\beta_{j}$ is the effect of the $j^{\text {th }}$ block, $l_{(i-1, j)}$ is the left neighbour effect due to the treatment in the $(i-1)^{\text {th }}$ plot of $j^{\text {th }}$ block, $\gamma_{(i+1, j)}$ is the right neighbour effect due to the treatment in the $(i+1)^{\text {th }}$ plot in $j^{\text {th }}$ block, $e_{i j}$ are error terms distributed with mean zero and a variance-covariance structure $\Omega=I_{b} \otimes \Lambda$ ( $I_{b}$ is an identity matrix of order $b$ and $\otimes$ denotes the kronecker product). Assuming no correlation among the observations between the blocks and correlation structure between plots within a block to be the same in each block, $\Lambda$ is the correlation matrix of $k$ observations within a block. If the errors within a block follow an Second Order Auto Regressive model $(\mathrm{AR}(2))$ then $\Omega=I_{b} \otimes M_{k}$ where $M_{k}$ is a $k \times k$ matrix given by

$$
M_{k}=\left[\begin{array}{ccccc}
r_{0} & r_{1} & r_{2} & \ldots & r_{k-1} \\
r_{1} & r_{0} & r_{1} & \ldots & r_{k-2} \\
\vdots & \vdots & \vdots & \vdots & \vdots \\
r_{k-1} & r_{k-2} & r_{k-3} & \ldots & r_{0}
\end{array}\right]
$$

The elements of $M_{k}$ are

$$
\begin{aligned}
& r_{0}=\left(1-\rho_{2}\right) /\left(1+\rho^{2}\right)\left\{\left(1-\rho_{2}\right)^{2}-\rho_{1}^{2}\right\} \\
& r_{1}=\left\{\rho_{1}^{2} /\left(1-\rho_{2}\right)\right\} r_{0} \\
& r_{2}=\left\{\rho_{1}^{2} /\left(1-\rho_{2}\right)+\rho_{2}\right\} r_{0}
\end{aligned}
$$


For $k \geq 3, r_{k}=\left\{\rho_{1} r_{k-1}+\rho_{2} r_{k-2}\right\} r_{0}$

If the errors within a block follow Second Order Moving Average model (MA(2)) the $\Omega=I_{b} \otimes N_{k}$ where $N_{k}$ is a $k \times k$ matrix given by

$$
N_{k}=\left[\begin{array}{cccccc}
1+\rho_{1}^{2}+\rho_{2}^{2} & \rho_{1}+\rho_{1} \rho_{2} & \rho_{2} & 0 & \ldots & 0 \\
\rho_{1}+\rho_{1} \rho_{2} & 1+\rho_{1}^{2}+\rho_{2}^{2} & \rho_{1}+\rho_{1} \rho_{2} & \rho_{2} & \ldots & 0 \\
\vdots & \vdots & \vdots & \vdots & \vdots & \vdots \\
\vdots & \vdots & \vdots & \vdots & \vdots & \vdots \\
\vdots & \vdots & \vdots & \vdots & \vdots & \vdots \\
0 & 0 & 0 & 0 & \ldots & 1+\rho_{1}^{2}+\rho_{2}^{2}
\end{array}\right]
$$

The NN correlation structure, the $\Lambda$ is a matrix with diagonal entries as 1 and off-diagonal entries as $\rho$. Model (2.1) can be rewritten in the matrix notation as follows:

$$
Y=\mu 1+\Delta^{\prime} \tau+\Delta_{1}^{\prime} l+\Delta_{2}^{\prime} \gamma+D^{\prime} \beta+e
$$

where $Y$ is $n \times 1$ vector of observations, 1 is $n \times 1$ vector of ones, $\Delta^{\prime}$ is an $n \times v$ incidence matrix of observations versus direct treatments, $\tau$ is $v \times 1$ vector of direct treatment effects, $\Delta_{1}^{\prime}$ is a $n \times v$ matrix of observations versus left neighbour treatment, $\Delta_{2}^{\prime}$ is a $n \times v$ matrix of observations versus right neighbour treatment, $l$ is $v \times 1$ vector of left neighbour effects, $\gamma$ is $v \times 1$ vector of right neighbour effects, $D^{\prime}$ is an $n \times b$ incidence matrix of observations versus blocks, $\beta$ is $b \times 1$ vector of block effects and $e$ is $n \times 1$ vector of errors. The joint information matrix for estimating the direct and neighbour (left and right) effects under correlated observations estimated by generalized least squares is obtained as follows:

$$
C=\left[\begin{array}{ccc}
\Delta\left(I_{b} \otimes \wedge^{*}\right) \Delta^{\prime} & \Delta\left(I_{b} \otimes \wedge^{*}\right) \Delta_{1}^{\prime} & \Delta\left(I_{b} \otimes \wedge^{*}\right) \Delta_{2}^{\prime} \\
\Delta_{1}\left(I_{b} \otimes \wedge^{*}\right) \Delta^{\prime} & \Delta_{1}\left(I_{b} \otimes \wedge^{*}\right) \Delta_{1}^{\prime} & \Delta_{1}\left(I_{b} \otimes \wedge^{*}\right) \Delta_{2}^{\prime} \\
\Delta_{2}\left(I_{b} \otimes \wedge^{*}\right) \Delta^{\prime} & \Delta_{2}\left(I_{b} \otimes \wedge^{*}\right) \Delta_{1}^{\prime} & \Delta_{2}\left(I_{b} \otimes \wedge^{*}\right) \Delta_{2}^{\prime}
\end{array}\right]
$$

with

$$
\wedge^{*}=\wedge^{-1}-\left(1_{k}^{\prime} \wedge^{-1} 1_{k}\right)^{-1} \wedge^{-1} 1_{k} 1_{k}^{\prime} \wedge^{-1}
$$

The above $3 v \times 3 v$ information matrix $(C)$ for estimating the direct effects and neighbour effects of treatments in a block design setting is symmetric, non-negative definite with row and column sums equal to zero. The information matrix for estimating the direct effects of treatments from (2.3) is as follows:

$$
C_{\tau}=C_{11}-C_{12} C_{22}^{-1} C_{21}
$$

where $\quad C_{11}=\Delta\left(I_{b} \otimes \wedge^{*}\right) \Delta^{\prime}$

$$
C_{12}=\left\lfloor\Delta\left(I_{b} \otimes \wedge^{*}\right) \Delta_{1}^{\prime} \quad \Delta\left(I_{b} \otimes \wedge^{*}\right) \Delta_{2}^{\prime}\right\rfloor
$$

and

$$
C_{22}=\left[\begin{array}{ll}
\Delta_{1}\left(I_{b} \otimes \wedge^{*}\right) \Delta_{1}^{\prime} & \Delta_{1}\left(I_{b} \otimes \wedge^{*}\right) \Delta_{2}^{\prime} \\
\Delta_{2}\left(I_{b} \otimes \wedge^{*}\right) \Delta_{1}^{\prime} & \Delta_{2}\left(I_{b} \otimes \wedge^{*}\right) \Delta_{2}^{\prime}
\end{array}\right]
$$

Similarly, the information matrix for estimating the left neighbour effect of treatments $\left(C_{l}\right)$ and right neighbour effect of treatments $\left(C_{\gamma}\right)$ can be obtained. 


\subsection{Construction of Design}

Tomer et al. [19] has constructed neighbour balanced block design with parameters $v$ (prime or prime power), $b=v(v-1), r=(v-1)(v-m), k=(v-m), \quad m=1,2, \ldots, v-4 \quad$ and $\quad \lambda=(v-m) \quad$ using Mutually Orthogonal Latin Squares (MOLS) of order $v$. This series of design bas been investigated under the correlated error structure. It is seen that the design turns out to be pair-wise uniform with $\alpha=1$ and also variance balanced for estimating direct $\left(V_{1}\right)$ and neighbour effects $\left(V_{2}=V_{3}\right)$.

\section{Example:}

Let $v=5$ and $m=0$. The following is a neighbour balanced pair-wise uniform complete block design with parameters $v=5, b=20, r=20, k=5, \lambda=5$ and $\alpha=1$ :

\begin{tabular}{l|lllll|l}
2 & 3 & 4 & 5 & 1 & 2 & 3 \\
3 & 4 & 5 & 1 & 2 & 3 & 4 \\
4 & 5 & 1 & 2 & 3 & 4 & 5 \\
5 & 1 & 2 & 3 & 4 & 5 & 1 \\
1 & 2 & 3 & 4 & 5 & 1 & 2 \\
3 & 4 & 5 & 1 & 2 & 3 & 4 \\
4 & 5 & 1 & 2 & 3 & 4 & 5 \\
5 & 1 & 2 & 3 & 4 & 5 & 1 \\
1 & 2 & 3 & 4 & 5 & 1 & 2 \\
2 & 3 & 4 & 5 & 1 & 2 & 3 \\
4 & 5 & 1 & 2 & 3 & 4 & 5 \\
5 & 1 & 2 & 3 & 4 & 5 & 1 \\
1 & 2 & 3 & 4 & 5 & 1 & 2 \\
2 & 3 & 4 & 5 & 1 & 2 & 3 \\
3 & 4 & 5 & 1 & 2 & 3 & 4 \\
5 & 1 & 2 & 3 & 4 & 5 & 1 \\
1 & 2 & 3 & 4 & 5 & 1 & 2 \\
2 & 3 & 4 & 5 & 1 & 2 & 3 \\
3 & 4 & 5 & 1 & 2 & 3 & 4 \\
4 & 5 & 1 & 2 & 3 & 4 & 5 \\
1 & 2 & 3 & 4 & 5 & 1 & 2 \\
2 & 3 & 4 & 5 & 1 & 2 & 3 \\
3 & 4 & 5 & 1 & 2 & 3 & 4 \\
4 & 5 & 1 & 2 & 3 & 4 & 5 \\
5 & 1 & 2 & 3 & 4 & 5 & 1
\end{tabular}

The information matrices for estimating the direct and neighbouring effects of treatments for AR(2) with $\rho=0.1\left(\rho_{1}=\rho_{2}=\rho\right)$ is obtained given as below:

$$
C_{\tau}=15.47944\left[I-\frac{J}{5}\right] \text { and } \quad C_{l}=C_{\gamma}=15.45525\left[I-\frac{J}{5}\right]
$$

Similarly for MA(2) \& NN structures,

$$
\begin{aligned}
& C_{\tau}=15.36396\left[I-\frac{J}{5}\right] \text { and } \quad C_{l}=C_{\gamma}=16.81641\left[I-\frac{J}{5}\right] \\
& C_{\tau}=9.12109\left[I-\frac{J}{5}\right] \text { and } \quad C_{l}=C_{\gamma}=10.44325\left[I-\frac{J}{5}\right]
\end{aligned}
$$

These matrices have been worked out using $\mathrm{R}$ package. For, $m=1$ the resulting design will be a neighbour balanced pair-wise uniform incomplete block design with parameters $v=5, b=20, r=16, k=4, \lambda=4$ and $\alpha=1$. 


\section{Efficiency of Neighbour Balanced Pair-Wise Uniform Block Designs:}

In this section, a quantitative measure of efficiency of the NNBD has been derived when error structure follows AR(2) and MA(2) models. The comparison of universally optimal neighbour balanced design for $v$ treatments in $(v-1)$ complete blocks of Azais et al. [3] considering observations to be correlated within the blocks. We compare the average variance of an elementary treatment contrast $\hat{\tau}_{s}-\hat{\tau}_{s^{\prime}}$ in both cases. The average variance of an elementary treatment contrast Kempthorne, [8] for direct effects of the neighbour balanced design of Azais et al. [3] estimated by generalized least squares methods, is given by

$$
V_{A}=\frac{2 \sigma^{2}}{v-1} \sum_{s=1}^{v-1} \theta_{s}^{-1}
$$

where $\theta_{s}$ 's are the $(v-1)$ non-zero eigen values of $C_{\tau}$ for Azais et al. [3], $\sigma^{2}$ is the variance of an observation. The efficiency factor $\left(E_{\tau}\right)$ for direct effects of the neighbour balanced pair-wise uniform block design is thus given as:

$$
E_{\tau}=\frac{(v-1) \sum_{s=1}^{v-1} \theta_{s}^{-1}}{(v-m) \sum_{s=1}^{v-1} \delta_{s}^{-1}}
$$

$\delta_{s}$ 's are the $(v-1)$ non-zero eigen values of $C_{\tau}$. Similarly the efficiency $\left(E_{l}\right)$ and $\left(E_{\gamma}\right)$ for neighbour effects (left and right) of treatments is obtained. The ranges of correlation coefficient $(\rho)$ for different correlation structures investigated are $|\rho| \leq 0.40$ for $\operatorname{AR}(2), \operatorname{MA}(2)$ and NN correlation structures. For these ranges, the matrix of correlation coefficients among observations within a block is positive definite. For $\rho=0$, the efficiency is that of totally balanced designs obtained by Tomer et al. [19].

In Tables 1, 2 and 3 the parameters of neighbour balanced pair-wise uniform block design for $v=5$ $(m=0)$ and $v=6(m=0,1,2)$ along with the efficiency for direct and neighbour effects (left and right) has been shown. The efficiency values have been reported under the AR(2), MA(2) and NN correlation structures with $\left(\rho_{1}, \rho_{2}\right)$ in the interval -0.4 (-0.4) 0.4. It is seen that efficiency for direct as well as neighbour effects is high, in case of complete block designs i.e., $(m=0)$ for NN correlation structure. In case of incomplete block designs $(m=1,2, \ldots, v-4) \mathrm{MA}(2)$ correlation structure turns out to be more efficient as compared to others models with $\left(\rho_{1}, \rho_{2}\right)$ in the interval 0.1 to 0.4 . It is therefore concluded that when block sizes are large and neighbouring plots are highly correlated, generalized least squares for estimation of direct and neighbour effects can be used.

\section{Efficiency of NNBD and NNBIBD in comparison to Regular Block Design}

In this section, a quantitative measure of efficiency of NNBD is also derived when the errors follow $\operatorname{AR}(2)$ and $\mathrm{MA}(2)$ models. If errors within a block follow an $\mathrm{AR}(2)$ then

$$
\begin{gathered}
V_{A R(2)}\left(\tau_{i}-\widehat{\tau}_{j}\right)=2 r^{-1} \sigma_{\varepsilon}^{2}, r_{0}\left[1+\frac{\alpha}{r_{0}}-\frac{2}{t(t-1)} \sum_{i=1}^{t-1}(t-i) r_{i}\right] \\
\text { where } \quad \begin{aligned}
r_{0}=\left(1-\rho_{2}\right) /\left(1+\rho_{2}\right)\left[\left(1-\rho_{2}\right)^{2}-\rho_{1}^{2}\right\rfloor \\
r_{1}=\left\{\rho_{1} /\left(1-\rho_{2}\right)\right\} r_{0} \\
r_{2}=\left\{\left\lfloor\rho_{1}^{2} /\left(1-\rho_{2}\right)\right]+\rho_{2}\right\} r_{0}
\end{aligned}
\end{gathered}
$$

for $k \geq 3, r_{k}=\left\{\rho_{1} r_{k-1}+\rho_{2} r_{k-2}\right\} r_{0}$ 
If errors follow second order moving average model MA(2), then

$$
\begin{gathered}
V_{M A(2)}\left(\tau_{i}-\widehat{\tau}_{j}\right)=2 r^{-1} \sigma_{\varepsilon}^{2}, r_{0}\left[1+\frac{\alpha}{r_{0}}-\frac{2}{t(t-1)} \sum_{i=1}^{t-1}(t-i) r_{i}\right] \\
\text { where, } \quad r_{0}=1+\rho_{1}^{2}+\rho_{2}^{2} \\
r_{1}=\rho_{1}+\rho_{1} \rho_{2} \\
r_{2}=\rho_{2}
\end{gathered}
$$

for $k \geq 3, r_{k}=0$ where $\alpha=\sigma_{\xi}^{2} / \sigma_{\varepsilon}^{2}$

For NNBD the variance of an elementary contrast is given by

$$
V_{N}=2(t-1)^{-1} \sum_{i=1}^{t-1} \gamma_{d i}^{-1}
$$

Where $\gamma_{d i}$ 's are non-zero values of $C_{d}$. We define the efficiency of a design $d$ relative to a regular block design as $\frac{V_{A R(2)}}{V_{N}}$ and $\frac{V_{M A(2)}}{V_{N}}$ respectively for AR(2) and MA(2) models.

The Tables 4, 5, 6 and 7 shows the efficiencies of NNBD with $t=5, r=20$ and $t=6, r=30$, $\left(\rho_{1}, \rho_{2}\right)=-0.4(-0.4) 0.4$ and $\alpha=1$. The values in the tables show that as $\rho$ increases from -0.4 to -0.1 the gain in efficiency also increases and $\rho$ decreases from 0.1 to 0.4 the gain in efficiency also decreases under $\mathrm{AR}(2)$ and MA(2) models. The gain in efficiency of NNBD over regular block design is high under MA(2) models ( $t=5, r=20$ and $\alpha=1$ ) for direct and neighbour effects of treatments.

The Tables 8, 9, 10, 11, 12 and 13 show the efficiencies of NNBIBD with $t=5, r=16, t=6, r=25$ and $t=6, r=20,\left(\rho_{1}, \rho_{2}\right)=-0.4(-0.4) 0.4$ and $\alpha=1$. The values in the tables show that as $\rho$ increases from -0.4 to -0.1 , the gain in efficiency also increases and $\rho$ decreases from 0.1 to 0.4 , the gain in efficiency also decreases under $\mathrm{AR}(2)$ and MA(2) models. The gain in efficiency of NNBIBD over regular block design is high under MA(2) models ( $t=6, r=20$ and $\alpha=1$ ) for direct and neighbour effects of treatments.

\section{Conclusion}

We have concluded that the efficiency for direct as well as neighbouring effects is high in the case of complete block designs for NN correlation structure. In the case of incomplete block designs, MA(2) correlation structure turned out to be more efficient as compared to other models with $\left(\rho_{1}, \rho_{2}\right)$ in the interval 0.1 to 0.4 . The gain in efficiency of NNBD and NNBIBD over regular block design is high under MA(2) models for direct and neighbour effects of treatments.

\section{References}

[1]. Anderson, A. H., Jensen, E.B., and Schou, G.: Two-way analysis of variance with correlated errors, Int. Statist. Reve. 49, 153-167 (1981).

[2]. Aastveit, A.H.: On the effect of correlation between plots in randomized block experiments. Biometrical J. 25, 129-153 (1983).

[3]. Azais, J.M., Bailey, R. A., and Monod, H.: A catalogue of efficient neighbour designs with border plots, Biometrics, 49, 1252-1261 (1993).

[4]. Box, G.E.P.: Some theorems of quadratic forms applied in the study of analysis of variance problems, II. Effects of inequality of variance and of correlation between errors in the two-way classification. Ann. Math. Statist. 25, 484-498 (1954).

[5]. Bailey, R. A.: Designs for one-sided neighbour effects, J. Ind. Soc. Agric. Statistics, 56(3), 302-314 (2003).

[6]. Gill, P. S. and Shukla, G. K.: Efficiency of nearest neighbour balanced block designs for correlated observations, Biometrika, 72, 539-544 (1985).

[7]. Iqbal I, Amanuallah M, Nasir J A.: The construction of second order neighbor designs, J. Res. (Science), BZU, Multan, 17(3), 191199 (2006).

[8]. Kempthorne, O.: The efficiency factor of an incomplete block design, Ann. Math. Statist., 27, 846-849 (1956).

[9]. Keedwell, A.D.: Circuit designs and latin squares. Ars Combinatoria, 17, 79-90 (1984).

[10]. Kunert, J.: Neighbour balanced block designs for correlated errors. Biometrika 74, 4, 717-724 (1987).

[11]. Kunert, J., Martin, R. J., and Pooladsaz, S.: Optimal designs under two related models for interference, Metrika, 57, 137-143 (2003).

[12]. Lindner, C.C., Phelps, K.T. and Rodger, C.A.: The spectrum for 2-perfect 6-cycles System, J. Combin. Theory, A 57, 76-85 (1991).

[13]. Morgan, J.P. and Nizamuddin.: Two dimensional design for correlated errors. The Ann. Of Statist. 19, 2160-2182 (1991). 
[14]. Martin, R. J. and Eccleston, J. A.: Variance-balanced designs under interference for dependent observations, J. Statist Plan \& Inference, 119, 207-223 (2004).

[15]. Rees, D.H. Some designs of use in serology, Biometrics, 23, 779-791 (1967).

[16]. R. Senthil Kumar, C. Santharam.: Efficiency of NNBD over NNBIBD using First Order Correlated Models, IOSR Journal of Mathematics (IOSR-JM), e-ISSN: 2278-5728, p-ISSN: 2319- 765X, Volume 7, Issue 5 (Jul. - Aug. 2013), PP 06-13 (2013).

[17]. R. Senthil Kumar \& C. Santharam.: Efficiency of Nearest Neighbour Balanced Block Designs using ARMA models, International Journal of Statistics and Systems, ISSN: 0973-2675 Volume 8, Number 1, pp. 59-71 (2013).

[18]. Santharam.C \& K.N.Ponnuswamy.: On the Efficiency of Nearest Neighbour Balanced Block Designs with Correlated Error Structure, Biometrics, J. 39, 85-98 (1997).

[19]. Tomar, J.S.Jaggi, S., and Varghese, C.: On totally balanced block designs for competition effects, Jour. Applied Statistics, 32 (1), 87-97 (2005).

[20]. Tomar, J.S.Jaggi, S.: Efficient neighbour balanced block designs for correlated observations, METRON - International Journal of Statistics, Vol. LXV, n. 2, pp. 229-238 (2007).

Tables:

Table 1. Efficiency of neighbour balanced pair-wise uniform block designs AR(2) model

\begin{tabular}{|c|c|c|c|c|c|c|c|c|}
\hline \multicolumn{5}{|c|}{ Parameters } & \multirow{2}{*}{\multicolumn{4}{|c|}{$\begin{array}{c}\text { Correlation Structure } \\
\mathbf{A R}(2)\end{array}$}} \\
\hline & & & & & & & & \\
\hline$v$ & $b$ & $m$ & $r$ & $k=\lambda$ & $\left(\rho_{1}, \rho_{2}\right)$ & $E_{\tau}$ & $E_{l}$ & $E_{\gamma}$ \\
\hline \multirow[t]{9}{*}{5} & 20 & 0 & 20 & 5 & $(-0.4,-0.4)$ & 0.56427 & 0.56808 & 0.54146 \\
\hline & & & & & $(-0.3,-0.3)$ & 0.64066 & 0.64761 & 0.64341 \\
\hline & & & & & $(-0.2,-0.2)$ & 0.78467 & 0.71141 & 0.60989 \\
\hline & & & & & $(-0.1,-0.1)$ & 0.76701 & 0.76929 & 0.76893 \\
\hline & & & & & $(0,0)$ & 0.80000 & 0.80000 & 0.80000 \\
\hline & & & & & $(0.1,0.1)$ & 0.80582 & 0.81691 & 0.79696 \\
\hline & & & & & $(0.2,0.2)$ & 0.79178 & 0.80068 & 0.80757 \\
\hline & & & & & $(0.3,0.3)$ & 0.72334 & 0.79433 & 0.73051 \\
\hline & & & & & $(0.4,0.4)$ & 0.88824 & 0.69126 & 0.70063 \\
\hline \multirow[t]{9}{*}{6} & 30 & 0 & 30 & 6 & $(-0.4,-0.4)$ & 0.60734 & 0.59666 & 0.58634 \\
\hline & & & & & $(-0.3,-0.3)$ & 0.68867 & 0.68532 & 0.73889 \\
\hline & & & & & $(-0.2,-0.2)$ & 0.74974 & 0.75752 & 0.76040 \\
\hline & & & & & $(-0.1,-0.1)$ & 0.80637 & 0.79854 & 0.80464 \\
\hline & & & & & $(0,0)$ & 0.83333 & 0.83333 & 0.83333 \\
\hline & & & & & $(0.1,0.1)$ & 0.85754 & 0.84921 & 0.84619 \\
\hline & & & & & $(0.2,0.2)$ & 0.83448 & 0.82871 & 0.88098 \\
\hline & & & & & $(0.3,0.3)$ & 0.81760 & 0.80621 & 0.76668 \\
\hline & & & & & $(0.4,0.4)$ & 0.72861 & 0.71027 & 0.68055 \\
\hline \multirow[t]{9}{*}{6} & 30 & 1 & 25 & 5 & $(-0.4,-0.4)$ & 0.83447 & 0.82390 & 0.81067 \\
\hline & & & & & $(-0.3,-0.3)$ & 0.87554 & 0.87287 & 0.87532 \\
\hline & & & & & $(-0.2,-0.2)$ & 0.92940 & 0.92201 & 0.93455 \\
\hline & & & & & $(-0.1,-0.1)$ & 0.97469 & 0.97316 & 0.98455 \\
\hline & & & & & $(0,0)$ & 1.00000 & 1.00000 & 1.00000 \\
\hline & & & & & $(0.1,0.1)$ & 1.03364 & 1.03548 & 1.04214 \\
\hline & & & & & $(0.2,0.2)$ & 1.04870 & 1.05409 & 1.04523 \\
\hline & & & & & $(0.3,0.3)$ & 1.03975 & 1.01356 & 1.01522 \\
\hline & & & & & $(0.4,0.4)$ & 0.94793 & 1.15515 & 1.70573 \\
\hline \multirow[t]{9}{*}{6} & 30 & 2 & 20 & 4 & $(-0.4,-0.4)$ & 0.93088 & 0.49412 & 0.87094 \\
\hline & & & & & $(-0.3,-0.3)$ & 1.03841 & 1.00006 & 1.00628 \\
\hline & & & & & $(-0.2,-0.2)$ & 1.13443 & 1.13032 & 1.13469 \\
\hline & & & & & $(-0.1,-0.1)$ & 1.21476 & 1.24003 & 1.23816 \\
\hline & & & & & $(0,0)$ & 1.25000 & 1.25000 & 1.25000 \\
\hline & & & & & $(0.1,0.1)$ & 1.32514 & 1.34697 & 1.32511 \\
\hline & & & & & $(0.2,0.2)$ & 1.38279 & 1.40407 & 1.41581 \\
\hline & & & & & $(0.3,0.3)$ & 1.38420 & 1.41755 & 1.40362 \\
\hline & & & & & $(0.4,0.4)$ & 1.43294 & 1.44810 & 1.42910 \\
\hline
\end{tabular}

Table 2. Efficiency of neighbour balanced pair-wise uniform block designs MA(2) model

\begin{tabular}{|c|c|c|c|c|c|c|c|c|}
\hline \multicolumn{5}{|c|}{ Parameters } & \multicolumn{4}{c|}{ Correlation Structure } \\
\hline$v$ & $b$ & $m$ & $r$ & $k=\lambda$ & $\left(\rho_{1}, \rho_{2}\right)$ & $E_{\tau}$ & $E_{l}$ & $E_{\gamma}$ \\
\hline 5 & 20 & 0 & 20 & 5 & $(-0.4,-0.4)$ & 0.50455 & 0.50991 & 0.49682 \\
\hline & & & & & $(-0.3,-0.3)$ & 0.57578 & 0.59003 & 0.58525 \\
\hline & & & & & $(-0.2,-0.2)$ & 0.71456 & 0.69540 & 0.65266 \\
\hline & & & & & $(-0.1,-0.1)$ & 0.73357 & 0.65061 & 0.74019 \\
\hline & & & & & $(0,0)$ & 0.80000 & 0.80000 & 0.80000 \\
\hline & & & & & $(0.1,0.1)$ & 0.85091 & 0.86773 & 0.84908 \\
\hline & & & & & $(0.2,0.2)$ & 0.86011 & 0.85519 & 0.83159 \\
\hline & & & & & $(0.3,0.3)$ & 0.83086 & 0.85669 & 0.82009 \\
\hline
\end{tabular}




\begin{tabular}{|c|c|c|c|c|c|c|c|c|}
\hline & & & & & $(0.4,0.4)$ & 0.75814 & 0.70742 & 0.74459 \\
\hline 6 & 30 & 0 & 30 & 6 & $(-0.4,-0.4)$ & 0.53221 & 0.52322 & 0.50447 \\
\hline & & & & & $(-0.3,-0.3)$ & 0.62195 & 0.61417 & 0.61648 \\
\hline & & & & & $(-0.2,-0.2)$ & 0.69486 & 0.66319 & 0.68810 \\
\hline & & & & & $(-0.1,-0.1)$ & 0.76892 & 0.76855 & 0.76305 \\
\hline & & & & & $(0,0)$ & 0.83333 & 0.83333 & 0.83333 \\
\hline & & & & & $(0.2,0.2)$ & 0.87748 & 0.87339 & 0.87662 \\
\hline & & & & & $(0.3,0.3)$ & 0.84813 & 0.81497 & 0.84059 \\
\hline & & & & & $(0.4,0.4)$ & 0.77341 & 0.62246 & 0.77547 \\
\hline 6 & 30 & 1 & 25 & 5 & $(-0.4,-0.4)$ & 0.66551 & 0.62619 & 0.65638 \\
\hline & & & & & $(-0.3,-0.3)$ & 0.74862 & 0.74420 & 0.75138 \\
\hline & & & & & $(-0.2,-0.2)$ & 0.84034 & 0.83530 & 0.83880 \\
\hline & & & & & $(-0.1,-0.1)$ & 0.92152 & 0.93806 & 0.93681 \\
\hline & & & & & $(0,0)$ & 1.00000 & 1.00000 & 1.00000 \\
\hline & & & & & $(0.1,0.1)$ & 1.06487 & 1.10040 & 1.07081 \\
\hline & & & & & $(0.2,0.2)$ & 1.12050 & 1.08714 & 1.11359 \\
\hline & & & & & $(0.3,0.3)$ & 1.13502 & 1.12539 & 1.12761 \\
\hline & & & & & $(0.4,0.4)$ & 1.12955 & 1.11200 & 1.10951 \\
\hline 6 & 30 & 2 & 20 & & $(-0.4,-0.4)$ & 0.79845 & 0.78892 & 0.79581 \\
\hline & & & & & $(-0.3,-0.3)$ & 0.86223 & 0.93426 & 0.88878 \\
\hline & & & & & $(-0.2,-0.2)$ & 1.05227 & 1.11215 & 1.06864 \\
\hline & & & & & $(-0.1,-0.1)$ & 1.15628 & 1.17838 & 1.23751 \\
\hline & & & & & $(0,0)$ & 1.25000 & 1.25000 & 1.25000 \\
\hline & & & & & $(0.1,0.1)$ & 1.35875 & 1.35286 & 1.41124 \\
\hline & & & & & $(0.2,0.2)$ & 1.42639 & 1.44671 & 1.47277 \\
\hline & & & & & $(0.3,0.3)$ & 1.46134 & 1.44887 & 1.43499 \\
\hline & & & & $(0.4,0.4)$ & 1.49548 & 1.42926 & 1.50805 \\
\hline
\end{tabular}

Table 3. Efficiency of neighbour balanced pair-wise uniform block designs NN model

\begin{tabular}{|c|c|c|c|c|c|c|c|c|}
\hline \multicolumn{3}{|c|}{ Parameters } & & \multicolumn{5}{c|}{ Correlation Structure } \\
\hline$v$ & $b$ & $m$ & $r$ & $k=\lambda$ & $\rho$ & $E_{\tau}$ & $E_{l}$ & $E_{\gamma}$ \\
\hline 5 & 20 & 0 & 20 & 5 & -0.4 & 0.57777 & 0.54370 & 0.55414 \\
\hline & & & & & -0.3 & 0.77230 & 0.76003 & 0.71921 \\
\hline & & & & & -0.2 & 1.12702 & 1.11899 & 1.12748 \\
\hline & & & & & -0.1 & 1.32548 & 1.27062 & 1.26528 \\
\hline & & & & & 0 & 0.80000 & 0.80000 & 0.80000 \\
\hline & & & & & 0.1 & 1.33665 & 1.39663 & 1.26119 \\
\hline & & & & & 0.2 & 1.16767 & 1.20560 & 1.13899 \\
\hline & & & & & 0.4 & 0.77862 & 0.78772 & 0.76156 \\
\hline 6 & 30 & 0 & 30 & 6 & -0.4 & 0.33727 & 0.36735 & 0.32629 \\
\hline & & & & & -0.3 & 0.46641 & 0.50243 & 0.45668 \\
\hline & & & & & -0.2 & 0.68408 & 0.68807 & 0.67801 \\
\hline & & & & & -0.1 & 1.37697 & 1.39017 & 1.34549 \\
\hline & & & & & 0 & 0.83333 & 0.83333 & 0.83333 \\
\hline & & & & & 0.1 & 1.44357 & 1.40101 & 1.40017 \\
\hline & & & & & 0.2 & 0.93128 & 0.90471 & 0.87864 \\
\hline & & & & & 0.3 & 0.62177 & 0.62254 & 0.58294 \\
\hline & & & & & 0.4 & 0.47132 & 0.45645 & 0.45121 \\
\hline 6 & 30 & 1 & 25 & 5 & -0.4 & 0.15597 & 0.21636 & 0.44046 \\
\hline & & & & & -0.3 & 0.24370 & 0.21980 & 0.49732 \\
\hline & & & & & -0.2 & 0.48998 & 0.41370 & 0.82479 \\
\hline & & & & & -0.1 & 0.90261 & 0.95073 & 0.95956 \\
\hline & & & & & 0 & 1.00000 & 1.00000 & 1.00000 \\
\hline & & & & & 0.1 & 1.01487 & 1.02528 & 1.13842 \\
\hline & & & & & 0.2 & 0.46956 & 0.55623 & 0.68783 \\
\hline & & & & & 0.3 & 0.38600 & 0.38782 & 0.34714 \\
\hline & 30 & 2 & 20 & 4 & -0.4 & 0.85435 & 0.90279 & 0.92465 \\
\hline & & & & & -0.3 & 1.08256 & 1.28288 & 1.18901 \\
\hline & & & & & -0.1 & 1.77180 & 1.65902 & 1.91532 \\
\hline & & & & & 0.1 & 1.25000 & 1.25000 & 1.25000 \\
\hline & & & & & 0.3 & 1.24573 & 1.30222 & 1.21305 \\
\hline & & & & & 0.4 & 0.94025 & 1.03260 & 0.77395 \\
\hline
\end{tabular}


Table 4. Efficiency of NNBD using AR(2) Model $(t=5, r=20$ and $\alpha=1)$

\begin{tabular}{|c|c|c|c|}
\hline$\left(\rho_{1}, \rho_{2}\right)$ & $E_{\tau}$ & $E_{l}$ & $E_{\gamma}$ \\
\hline$(-0.4,-0.4)$ & 0.99707 & 0.94537 & 1.02110 \\
\hline$(-0.3,-0.3)$ & 1.10683 & 1.09303 & 1.05162 \\
\hline$(-0.2,-0.2)$ & 1.26307 & 1.40307 & 1.66570 \\
\hline$(-0.1,-0.1)$ & 2.58826 & 2.55587 & 2.57687 \\
\hline$(0,0)$ & 0.83954 & 0.85159 & 0.86020 \\
\hline$(0.1,0.1)$ & 2.42797 & 2.49175 & 2.43415 \\
\hline$(0.2,0.2)$ & 1.25211 & 1.29677 & 1.22696 \\
\hline$(0.3,0.3)$ & 0.93993 & 0.87836 & 0.93270 \\
\hline$(0.4,0.4)$ & 0.49401 & 0.30393 & 0.62504 \\
\hline
\end{tabular}

Table 5. Efficiency of NNBD using AR(2) Model $(t=6, r=30$ and $\alpha=1)$

\begin{tabular}{|c|c|c|c|}
\hline$\left(\rho_{1}, \rho_{2}\right)$ & $E_{\tau}$ & $E_{l}$ & $E_{\gamma}$ \\
\hline$(-0.4,-0.4)$ & 0.53382 & 0.54339 & 0.54519 \\
\hline$(-0.3,-0.3)$ & 0.61517 & 0.61818 & 0.57215 \\
\hline$(-0.2,-0.2)$ & 0.79475 & 0.78659 & 0.79152 \\
\hline$(-0.1,-0.1)$ & 1.44493 & 1.43084 & 1.44203 \\
\hline$(0,0)$ & 0.83238 & 0.83238 & 0.84832 \\
\hline$(0.1,0.1)$ & 1.78390 & 1.80140 & 1.77907 \\
\hline$(0.2,0.2)$ & 0.93644 & 0.94297 & 0.85290 \\
\hline$(0.3,0.3)$ & 0.64644 & 0.65558 & 0.65871 \\
\hline$(0.4,0.4)$ & 0.35792 & 0.36716 & 0.37387 \\
\hline
\end{tabular}

Table 6. Efficiency of NNBD using MA(2) Model $(t=5, r=20$ and $\alpha=1)$

\begin{tabular}{|c|c|c|c|}
\hline$\left(\rho_{1}, \rho_{2}\right)$ & $E_{\tau}$ & $E_{l}$ & $E_{\gamma}$ \\
\hline$(-0.4,-0.4)$ & 1.21164 & 1.14440 & 1.20920 \\
\hline$(-0.3,-0.3)$ & 1.30915 & 1.27530 & 1.22896 \\
\hline$(-0.2,-0.2)$ & 1.44002 & 1.49026 & 1.61606 \\
\hline$(-0.1,-0.1)$ & 2.15184 & 2.07303 & 2.12199 \\
\hline$(0,0)$ & 0.83954 & 0.85159 & 0.86020 \\
\hline$(0.1,0.1)$ & 2.26616 & 2.31200 & 2.25180 \\
\hline$(0.2,0.2)$ & 1.17302 & 1.17594 & 1.15405 \\
\hline$(0.3,0.3)$ & 0.77541 & 0.77173 & 0.78728 \\
\hline$(0.4,0.4)$ & 0.64231 & 0.76367 & 0.65257 \\
\hline
\end{tabular}

Table 7. Efficiency of NNBD using MA(2) Model $(t=6, r=30$ and $\alpha=1)$

\begin{tabular}{|c|c|c|c|}
\hline$\left(\rho_{1}, \rho_{2}\right)$ & $E_{\tau}$ & $E_{l}$ & $E_{\gamma}$ \\
\hline$(-0.4,-0.4)$ & 0.66202 & 0.74614 & 0.68862 \\
\hline$(-0.3,-0.3)$ & 0.72312 & 0.80247 & 0.72799 \\
\hline$(-0.2,-0.2)$ & 0.88883 & 0.95291 & 0.90663 \\
\hline$(-0.1,-0.1)$ & 1.53897 & 1.58135 & 1.54437 \\
\hline$(0,0)$ & 0.83238 & 0.84678 & 0.84832 \\
\hline$(0.1,0.1)$ & 1.77126 & 1.72860 & 1.73183 \\
\hline$(0.2,0.2)$ & 0.86914 & 0.86297 & 0.83654 \\
\hline$(0.3,0.3)$ & 0.62122 & 0.64246 & 0.58433 \\
\hline$(0.4,0.4)$ & 0.51164 & 0.62631 & 0.49786 \\
\hline
\end{tabular}

Table 8. Efficiency of NNBIBD using AR(2) Model $(t=5, r=16$ and $\alpha=1)$

\begin{tabular}{|c|c|c|c|}
\hline$\left(\rho_{1}, \rho_{2}\right)$ & $E_{\tau}$ & $E_{l}$ & $E_{\gamma}$ \\
\hline$(-0.4,-0.4)$ & 0.18527 & 0.28995 & 0.26233 \\
\hline$(-0.3,-0.3)$ & 0.28763 & 0.12989 & 0.16658 \\
\hline$(-0.2,-0.2)$ & 0.37600 & 0.37649 & 0.18700 \\
\hline$(-0.1,-0.1)$ & 0.71467 & 0.70473 & 0.62399 \\
\hline$(0,0)$ & 0.35740 & 0.35740 & 0.35740 \\
\hline$(0.1,0.1)$ & 0.72382 & 0.66743 & 0.75656 \\
\hline$(0.2,0.2)$ & 0.34895 & 0.35068 & 0.35801 \\
\hline$(0.3,0.3)$ & 0.29492 & 0.30014 & 0.29630 \\
\hline$(0.4,0.4)$ & 0.16895 & 0.12241 & 0.11859 \\
\hline
\end{tabular}


Table 9. Efficiency of NNBIBD using AR(2) Model $(t=6, r=25$ and $\alpha=1)$

\begin{tabular}{|c|c|c|c|}
\hline$\left(\rho_{1}, \rho_{2}\right)$ & $E_{\tau}$ & $E_{l}$ & $E_{\gamma}$ \\
\hline$(-0.4,-0.4)$ & 0.07397 & 0.01262 & 0.13114 \\
\hline$(-0.3,-0.3)$ & 0.10149 & 0.11443 & 0.13766 \\
\hline$(-0.2,-0.2)$ & 0.18434 & 0.16134 & 0.07707 \\
\hline$(-0.1,-0.1)$ & 0.31455 & 0.23519 & 0.27502 \\
\hline$(0,0)$ & 0.33414 & 0.33164 & 0.33772 \\
\hline$(0.1,0.1)$ & 0.32705 & 0.31905 & 0.31155 \\
\hline$(0.2,0.2)$ & 0.15082 & 0.15102 & 0.15221 \\
\hline$(0.3,0.3)$ & 0.12668 & 0.12051 & 0.13172 \\
\hline$(0.4,0.4)$ & 0.07168 & 0.05185 & 0.03884 \\
\hline
\end{tabular}

Table 10. Efficiency of NNBIBD using AR(2) Model $(t=6, r=20$ and $\alpha=1)$

\begin{tabular}{|c|c|c|c|}
\hline$\left(\rho_{1}, \rho_{2}\right)$ & $E_{\tau}$ & $E_{l}$ & $E_{\gamma}$ \\
\hline$(-0.4,-0.4)$ & 0.52996 & 0.71555 & 0.59596 \\
\hline$(-0.3,-0.3)$ & 0.56881 & 0.67374 & 0.62672 \\
\hline$(-0.2,-0.2)$ & 0.81717 & 0.56405 & 0.85855 \\
\hline$(-0.1,-0.1)$ & 1.50430 & 1.51538 & 0.36159 \\
\hline$(0,0)$ & 0.48303 & 0.48130 & 0.48606 \\
\hline$(0.1,0.1)$ & 1.33171 & 0.99505 & 1.42879 \\
\hline$(0.2,0.2)$ & 0.67638 & 0.69771 & 0.64563 \\
\hline$(0.3,0.3)$ & 0.45953 & 0.45152 & 0.42899 \\
\hline$(0.4,0.4)$ & 0.11869 & 0.08814 & 0.09162 \\
\hline
\end{tabular}

Table 11. Efficiency of NNBIBD using MA(2) $\operatorname{Model}(t=5, r=16$ and $\alpha=1)$

\begin{tabular}{|c|c|c|c|}
\hline$\left(\rho_{1}, \rho_{2}\right)$ & $E_{\tau}$ & $E_{l}$ & $E_{\gamma}$ \\
\hline$(-0.4,-0.4)$ & 0.25723 & 0.38273 & 0.35743 \\
\hline$(-0.3,-0.3)$ & 0.41218 & 0.16191 & 0.23084 \\
\hline$(-0.2,-0.2)$ & 0.43682 & 0.43746 & 0.22084 \\
\hline$(-0.1,-0.1)$ & 0.80030 & 0.74586 & 0.70076 \\
\hline$(0,0)$ & 0.35746 & 0.35746 & 0.35746 \\
\hline$(0.1,0.1)$ & 0.67062 & 0.65268 & 0.70637 \\
\hline$(0.2,0.2)$ & 0.31053 & 0.30814 & 0.31343 \\
\hline$(0.3,0.3)$ & 0.25911 & 0.24575 & 0.24123 \\
\hline$(0.4,0.4)$ & 0.14553 & 0.14651 & 0.14770 \\
\hline
\end{tabular}

Table 12. Efficiency of NNBIBD using MA(2) Model $(t=6, r=25$ and $\alpha=1)$

\begin{tabular}{|c|c|c|c|}
\hline$\left(\rho_{1}, \rho_{2}\right)$ & $E_{\tau}$ & $E_{l}$ & $E_{\gamma}$ \\
\hline$(-0.4,-0.4)$ & 0.10080 & 0.01805 & 0.17602 \\
\hline$(-0.3,-0.3)$ & 0.12601 & 0.14248 & 0.17024 \\
\hline$(-0.2,-0.2)$ & 0.21132 & 0.18459 & 0.08900 \\
\hline$(-0.1,-0.1)$ & 0.33790 & 0.24780 & 0.29348 \\
\hline$(0,0)$ & 0.33414 & 0.33414 & 0.33414 \\
\hline$(0.1,0.1)$ & 0.31362 & 0.29660 & 0.29955 \\
\hline$(0.2,0.2)$ & 0.13776 & 0.14291 & 0.13943 \\
\hline$(0.3,0.3)$ & 0.11287 & 0.10556 & 0.11534 \\
\hline$(0.4,0.4)$ & 0.09128 & 0.08172 & 0.09062 \\
\hline
\end{tabular}

Table 13. Efficiency of NNBIBD using MA(2) Model $(t=6, r=20$ and $\alpha=1)$

\begin{tabular}{|c|c|c|c|}
\hline$\left(\rho_{1}, \rho_{2}\right)$ & $E_{\tau}$ & $E_{l}$ & $E_{\gamma}$ \\
\hline$(-0.4,-0.4)$ & 0.67145 & 0.69124 & 0.70874 \\
\hline$(-0.3,-0.3)$ & 0.72723 & 0.76561 & 0.75328 \\
\hline$(-0.2,-0.2)$ & 0.91314 & 0.59420 & 0.94491 \\
\hline$(-0.1,-0.1)$ & 1.60506 & 1.61956 & 0.36742 \\
\hline$(0,0)$ & 0.48303 & 0.48130 & 0.48606 \\
\hline$(0.1,0.1)$ & 1.28310 & 0.78202 & 1.32538 \\
\hline$(0.2,0.2)$ & 0.63993 & 0.66086 & 0.60573 \\
\hline$(0.3,0.3)$ & 0.42334 & 0.42966 & 0.40811 \\
\hline$(0.4,0.4)$ & 0.33980 & 0.37711 & 0.25161 \\
\hline
\end{tabular}

